\title{
Design of an Amorphous In-Ga-Zn-O TFT Current-Scaling Pixel Driving Circuit for AMOLED with Threshold-voltage Shift Compensating
}

\author{
Chuncheng Yang, Xin Chen, Jian Zhang, Chuannan Li* \\ State Key Laboratory on Integrated Optoelectronics, College of Electronic Science and Engineering, Jilin University, 2699 \\ Qianjin Street, Changchun 130012, China \\ * e-mail: licn@jlu.edu.cn
}

\begin{abstract}
An Amorphous In-Ga-Zn-O TFT (a-IGZO TFTs) Current-Scaling Pixel Circuit for Active Matrix Organic Light-emitting Displays (AMOLEDs) is presented, it composes of four amorphous a-IGZO TFTs and one capacitor (4T-1C), which effectively compensates the threshold-voltage-shift $(\Delta V t h \approx 1 V)$ of the drive TFT as well as degradation of the OLED. In a monochromatic 2-inches QVGA AMOLED display, simulating results show that programming current between several pA and hundreds of $n A$ can realize OLED luminance from $1 \mathrm{~cd} / \mathrm{m}^{2}$ to $200 \mathrm{~cd} / \mathrm{m}^{2}$, and the aperture ratio of AMOLED with this pixel driving circuit is high as $80 \%$.
\end{abstract}

Keywords-AMOLED; a-IGZO-TFT; threshold-voltage-shift; pixel circuit;

\section{INTRODUCTION}

Organic Light-emitting Displays (OLEDs) are promised to be next generation of flat panel displays (FPDs) due to its perfect characteristics such as low power consumption, fast response, high contrast ratio, broad color range, wide viewing angle and thin display module etc. [1-4]. Active Matrix OLEDs (AMOLEDs), which consists of a thin film transistor (TFT) array as a series of switches to control the current flowing to each individual pixel, provide higher refresh rates than the passive-matrix OLED counterparts, improving response time often to under a millisecond, and AMOLEDs consume significantly less power. This advantage makes AMOLEDs well suited for portable electronics, where power consumption is critical to battery life. In addition, it has been proved to be feasible to convert an existing AMLCD line to an AMOLED production line, and the cost is only $40 \%$ of that for ramping up a new one [5]. Hydrogenated amorphous silicon TFTs (a-Si:H TFTs) and low temperature polycrystalline silicon TFTs (LTPS TFTs) currently are all used as the driving circuits for FPDs. Compared with LTPS TFTs, a-Si:H TFTs is more uniform for large area AMOLEDs, and its technology is mature and low cost, but its mobility is low (about $1 \mathrm{~cm}^{2} / \mathrm{Vs}$ ), on the other side LTPS TFTs have the advantage of high field-effect mobility $\left(>50 \mathrm{~cm}^{2} / \mathrm{Vs}\right)$. The low field-effect mobility of a-Si:H TFTs and non-uniformity of LTPS TFTs limit their applications for FPDs in future [6,7]. Recently, amorphous In-Ga-Zn-O thin-film transistor (a-IGZO TFT) have gained special attentions as an alternative to a-Si:H TFTs and LTPS TFTs due to its good uniformity, higher mobility $\left(>10 \mathrm{~cm}^{2} / \mathrm{Vs}\right)$, low leakage current, low processing temperature, visible transparency, sharp sub-threshold swing and potentially better electrical stability [8-11], which make it very attractive for the AMOLEDs driving circuits.

Here a current-scaling pixel circuit composed of four a-IGZO TFTs and one capacitor is presented, which usually is called 4T-1C a-IGZO TFTs pixel circuit, and it could be applied to monochromatic 2-inches QVGA (Quarter Video Graphics Array) AMOLEDs. The proposed pixel circuit is programmed with a current input signal and effectively compensates threshold-voltage-shift of the drive TFT and the degradation of OLED, compared with the conventional 2T-1C a-IGZO TFTs pixel circuit in which the driving current on OLED will be reduced because the threshold voltage of the driving TFT and the turn-on voltage of OLED all increase with the operating time. This 4T-1C a-IGZO TFTs pixel circuit has a high aperture ratio of circuit as $80 \%$, and 16 gray-scales of AMOLED with the luminance between $1 \mathrm{~cd} / \mathrm{m}^{2}$ and $200 \mathrm{~cd} / \mathrm{m}^{2}$ is achieved too.

\section{4T-1C a-IGZO TFTs PiXel CiRCUIT}

It is well known that the luminance of OLED is proportional to its driving current, Fig. 1 shows the luminance versus current characteristic $(\mathrm{L} \sim \mathrm{I})$ of the bottom-emitting OLED (BEOLED), and pixel area $\left(\mathrm{S}_{\text {pixel }}\right)$ for a 2-inches QVGA display is about $120 \mu \mathrm{m} \times 120 \mu \mathrm{m}$. Both voltage-scaling and current-scaling pixel driving circuit can be used for AMOLEDs, Fig. 2 (a) shows a current-scaling pixel circuit which is programmed with a current input signal, it composed of four a-IGZO TFTs T1 T4 and a storage capacitor, in which $\mathrm{T} 4$ is used as the driving transistor, T1 and T2 as switches and T3 as the threshold voltage compensating transistor, the diode represents the luminescent unit OLED in each pixel.

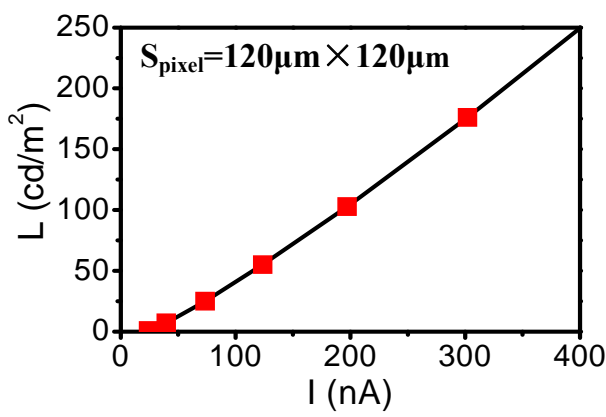

Figure 1. Luminance versus current curve of BEOLED. 


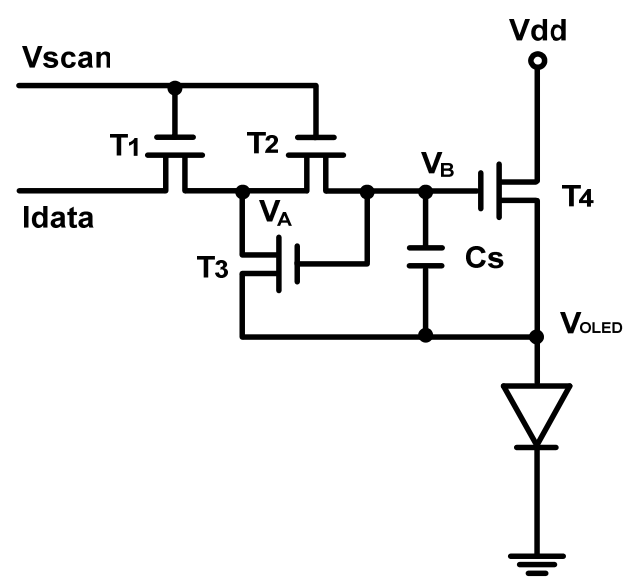

(a)

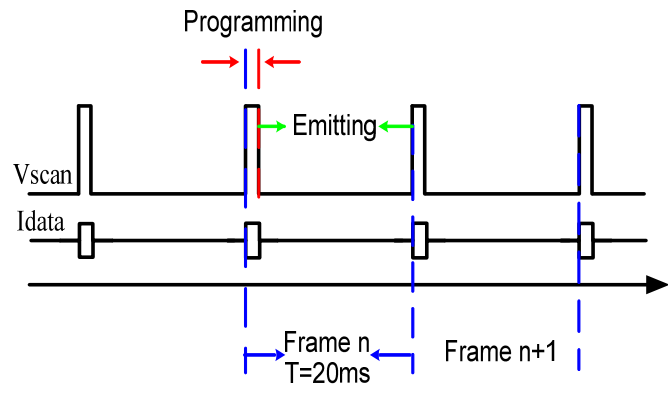

(b)

Figure 2. (a) Schematic of the 4T-1C a-IGZO TFTs pixel circuit,

(b) Control-signal timing diagram of the proposed pixel circuit.

The proposed pixel circuit operates in two periods in each frame, as shown in Fig. 2(b). The first stage is data programming period, during this stage $\mathrm{V}_{\text {scan }}$ is high so switching TFTs T1 and T2 are turned on, input current $I_{\text {data }}$ charge up $\mathrm{Cs}$ and $\mathrm{V}_{\mathrm{B}}$ at the $\mathrm{T} 3$ and $\mathrm{T} 4$ gate increases. When the voltage of $\mathrm{Cs}\left(\mathrm{V}_{\mathrm{Cs}}\right)$ become higher than the threshold-voltage of $\mathrm{T} 3$ and $\mathrm{T} 4\left(\mathrm{~V}_{\text {th3 }}\right.$ and $\left.\mathrm{V}_{\text {th }}\right)$, they are turned on and the current of both $\mathrm{T} 3$ and $\mathrm{T} 4\left(\mathrm{I}_{\mathrm{T} 3}\right.$ and $\left.\mathrm{I}_{\mathrm{T} 4}\right)$ grow. Current from T4 drive OLED in the pixel and current of T3 reduce the charging current of Cs, finally the charging to Cs finish until $\mathrm{I}_{\mathrm{T} 3}$ is equal to $\mathrm{I}_{\text {data, }}$, which is the end of this data programming period, see (1) and (2). At this moment, $V_{B}$ is a constant related to $I_{\text {data. }}$. During the second period, voltage $\mathrm{V}_{\text {scan }}$ becomes low and $\mathrm{T} 1 \sim \mathrm{T} 2$ are turned off to maintain the $\mathrm{V}_{\mathrm{Cs}}$ of $\mathrm{Cs}$, therefore $\mathrm{I}_{\mathrm{T} 4}$ and the current of OLED ( $\left.\mathrm{I}_{\mathrm{OLED}}\right)$ would be a constant until the next data is written in.

$$
\begin{gathered}
\mathrm{I}_{\text {data }}=\mathrm{I}_{\mathrm{T} 3}=\frac{1}{2} \times \mathrm{k} \times\left(\frac{\mathrm{W}}{\mathrm{L}}\right)_{3} \times\left(\mathrm{V}_{\mathrm{B}}-\mathrm{V}_{\mathrm{OLED}}-\mathrm{V}_{\mathrm{th} 3}\right)^{2} \\
\mathrm{I}_{\mathrm{OLED}}=\mathrm{I}_{\mathrm{T} 4}=\frac{1}{2} \times \mathrm{k} \times\left(\frac{\mathrm{W}}{\mathrm{L}}\right)_{4} \times\left(\mathrm{V}_{\mathrm{B}}-\mathrm{V}_{\mathrm{OLED}}-\mathrm{V}_{\mathrm{th} 4}\right)^{2}
\end{gathered}
$$

TFTs in the same driving circuit are fabricated simultaneously during the same fabrication process simultaneously, and the voltage $\mathrm{V}_{\mathrm{B}}$ at the gate of T3 and T4 is always same, then $\mathrm{V}_{\text {th } 3}$ and $\mathrm{V}_{\text {th4 }}$ are considered equal approximately and both will be increased with the operating time [12]. The turn-on voltage of OLED ( $\mathrm{V}_{\text {OLED }}$ ) also increases over the operating time. From (1)\&(2) and the mechanism of the 4T-1C a-IGZO TFTs pixel driving circuit, we know that $\mathrm{I}_{\mathrm{OLED}}$ is only decided by the size ratio between $\mathrm{T} 3$ and $\mathrm{T} 4$ and the programming current $\mathrm{I}_{\mathrm{data}}$, as shown in (3), hence the threshold-voltage-shift of driving TFT and degradation of OLED would be compensated.

$$
\frac{\mathrm{I}_{\mathrm{OLED}}}{\mathrm{I}_{\text {data }}}=\frac{(\mathrm{W} / \mathrm{L})_{4}}{(\mathrm{~W} / \mathrm{L})_{3}}
$$

\section{EQuivalent Model OF OLED}

In the 4T-1C a-IGZO TFTs pixel driving circuit the diode represents the luminescent unit OLED in each pixel, in fact a dual diode paralleled model is adopted as the equivalent circuit of BEOLED used during simulation, shown in Fig. 3. The maximum error $(<10 \%)$ between experiment data and the fitted data is tolerable. The fitted parameters of equivalent model are shown in Table I. Where $I_{s}$ is the reverse saturation current of diode, $n$ is the Non-ideological factor and $r_{s}$ is the series resistance.

\section{Hspice Simulation Result}

To detect the effect of the proposed pixel circuit, simulation using HSPICE was performed. The a-IGZO TFT HSPICE model was developed from the LEVEL 61 Rensselaer Polytechnic Institute (RPI) a-Si:H TFT model [13].

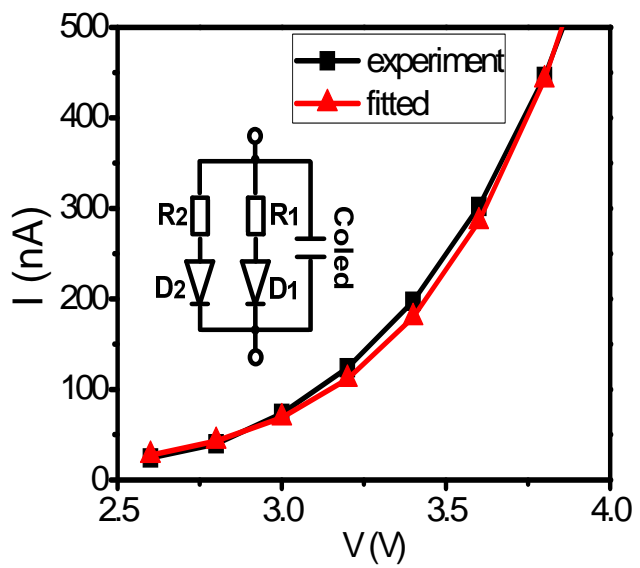

Figure 3. OLED equivalent model and experiental vs fitted curve.

TABLE I. BEOLED HSPICE PARAMETERS

\begin{tabular}{|c|c|}
\hline Parameter & Value \\
\hline Area & $120 \mu \mathrm{m} \times 120 \mu \mathrm{m}$ \\
\hline Coled & $2.704 \mathrm{pF}$ \\
\hline R1 & $3.2 \times 10^{4} \Omega$ \\
\hline R2 & $2.4 \times 10^{5} \Omega$ \\
\hline D1 & $\mathrm{Is}=8.0 \times 10^{-3} \mathrm{~A}, \mathrm{n}=50, \mathrm{r}_{\mathrm{s}}=0$, level $=1$ \\
\hline D2 & $\mathrm{Is}=1.0 \times 10^{-4} \mathrm{~A}, \mathrm{n}=14, \mathrm{r}_{\mathrm{s}}=0$, level $=1$ \\
\hline
\end{tabular}


The scan time (programming time) of each row is about $83 \mu$ s when frame frequency is $50 \mathrm{~Hz}$, the data programming time must be short than $83 \mu$ s, thus the value of Cs shouldn't be too large. But a small storage capacitor Cs also impacts the stability of Vcs after programming due to the leakage current of switching TFTs $\mathrm{T} 1 \& \mathrm{~T} 2$. Here $\mathrm{Cs}=0.5 \mathrm{pF}$ was selected and it exists

$$
\mathrm{C}_{\mathrm{S}}=\varepsilon_{0} \cdot \varepsilon_{\mathrm{r}} \times \mathrm{S} / \mathrm{d}_{0}
$$

Where $\mathrm{d}_{0}$ is the thickness of oxide layer of Cs, if $\mathrm{d}_{0}=100 \mathrm{~nm}$, the area of Cs would be about $1450 \mu \mathrm{m}^{2}$. Table II shows parameters used in HSPICE simulation, the aperture ratio of the proposed circuit with parameters shown in Table II is higher to $80 \%$. Fig. 4 shows the HSPICE simulation results of $\mathrm{I}_{\mathrm{OLED}}$ and $\mathrm{I}_{\text {data }}$ while the threshold-voltage-shift of driving TFT is $0 \mathrm{~V}, 0.5 \mathrm{~V}$ and $1 \mathrm{~V}$.

From Fig. 4, we know that the deviation is little even when $\Delta \mathrm{V}_{\mathrm{th}}=1 \mathrm{~V}$, therefore the proposed pixel circuit has the capability to compensate for threshold-voltage-shift of drive TFT which is helpful to improve the luminescent stability and uniformity of OLED. Furthermore, a smaller current (several $\mathrm{nA}$ ) could be realized even though the writing data $\mathrm{I}_{\text {data }}$ is larger (hundreds $\mathrm{nA}$ ), which can shorten the charging time at low gray-scale significantly. When the luminance of OLED is between $1 \mathrm{~cd} / \mathrm{m}^{2}$ and $200 \mathrm{~cd} / \mathrm{m}^{2}$, the simulation result of $\mathrm{I}_{\text {oled }}$ and $\mathrm{V}_{\mathrm{Cs}}\left(\mathrm{V}_{\mathrm{B}}-\mathrm{V}_{\text {OLED }}\right)$ are shown in Fig. 5.

TABLE II. PARAMETERS USED IN HSPICE SimULATION

\begin{tabular}{|c|c|}
\hline parameters & value \\
\hline T1 T2 T4 & $30 \mu \mathrm{m} / 30 \mu \mathrm{m}$ \\
\hline $\mathrm{T} 3$ & $300 \mu \mathrm{m} / 30 \mu \mathrm{m}$ \\
\hline Cs & $0.5 \mathrm{pF}$ \\
\hline Vdd & $15 \mathrm{~V}$ \\
\hline Vscan & $0 \rightarrow 15 \mathrm{~V}$ \\
\hline Idata & $290 \mathrm{nA} \rightarrow 4105 \mathrm{nA}$ \\
\hline
\end{tabular}

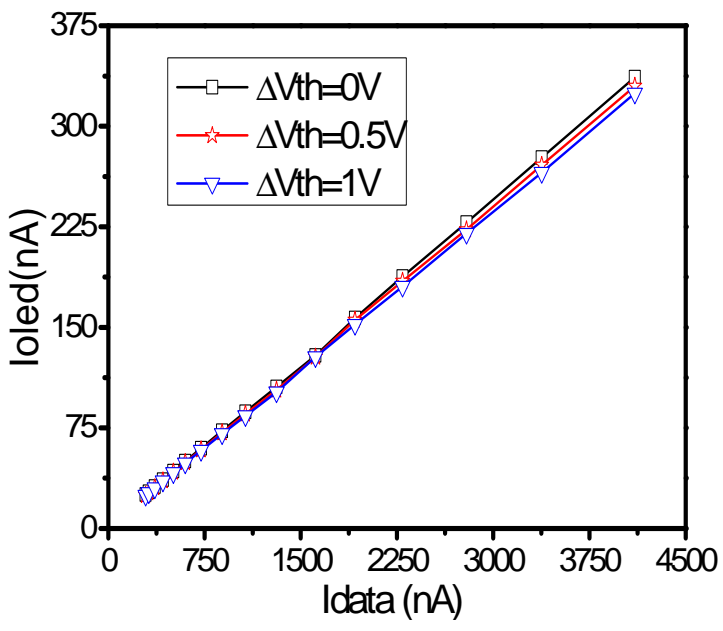

Figure 4. Simulated Ioled and Idata with different Vth shifts.

\section{CONCLUSION}

A current-scaling pixel circuit using four a-IGZO TFTs and one storage capacitor is proposed in the paper, its mechanism and the simulation results show that it can compensate the threshold-voltage-shift of drive TFT and the degradation of OLED effectively, and the aperture ratio of AMOLED applied this circuit is high to $80 \%$. At the same time, 16 gray-scales of AMOLED have been realized accurately corresponding to the luminance of OLED between $1 \mathrm{~cd} / \mathrm{m}^{2}$ and $200 \mathrm{~cd} / \mathrm{m}^{2}$.

\section{ACKNOWLEDGMENT}

The authors would like to thank the Ministry of Science and Technology of China (Grant No.2010CB327701), and the Foundation of Jilin Research Council (Grant No. 20090356) for financial support.
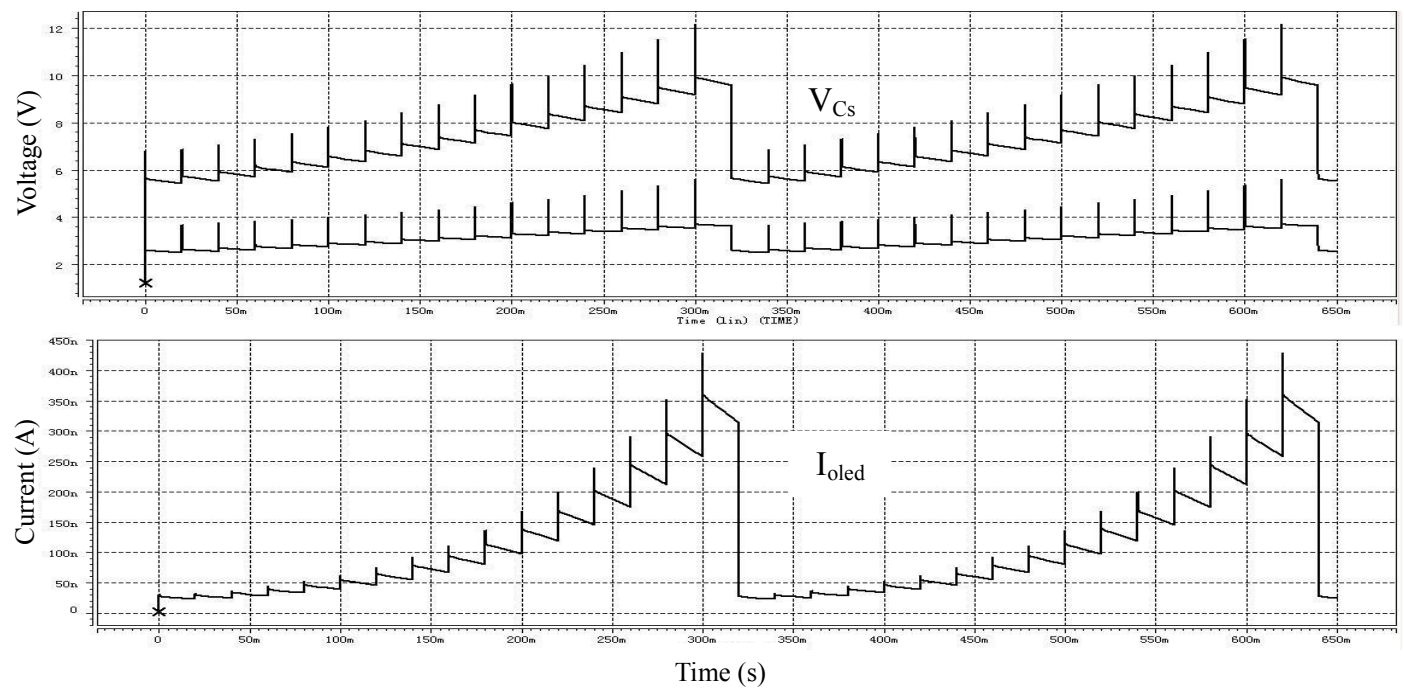

Figure 5. The simulation result of $\mathrm{I}_{\text {oled }}$ and $\mathrm{V}_{\mathrm{Cs}}$ with 16 gray-scales. 


\section{REFERENCES}

[1] C. W. Tang, "An overview of organic electro-luminescent materials and devices," J. Soc. Inf. Disp. Vol. 5, no.1, pp. 11-14, Mar 1997.

[2] Urabe Tetsuo, "The outstanding potential of OLED displays for TV applications," Inf. Disp. Vol. 24, no. 9, pp. 14-17, Sept 2008.

[3] AM-OLED-Tv Samsung versus LG-Display technical background http://www.oled-display.net/am-oled-tv-samsung-versus-lg-display-t echnical-background/, Mar 2012.

[4] AUO is ready to mass produce high resolution AM OLEDs OLED-TV samples end $2012 \mathrm{http} / / \mathrm{www}$.oled-display.net/auo-isready-to-mass-produce-high-resolution-amoleds-oled-tv-samples-end -2012/, Feb 2012.

[5] LG Display mass production of 55 inch OLED-Tv can start end 2013 http://www.oled-display.net/lg-display-mass-production-of-55-inch-o led-tv-can-start-end-2013/, Jan 2012.

[6] Juhn Suk Yoo, Hojin Lee, Jerzy Kanicki, Chang-Dong Kim, In-Jae Chung, "Novel a-Si:H TFT pixel circuit for electrically stable top-anode light-emitting AMOLEDs," J. Soc. Inf. Disp. Vol. 15, no. 8, pp. 545-551, Aug 2007.

[7] Kenji Nomura, Hiromichi Ohta, Akihiro Takagi, Toshio Kamiya,, Masahiro Hirano and Hideo Hosono, "Room-temperature fabrication of transparent flexible thin-film transistors using amorphous oxide semiconductors," Nature 432, pp. 488-492, Nov 2004.
[8] Toshio Kamiya, Kenji Nomura and Hideo Hosono, "Present status of amorphous In-Ga-Zn-O thin-film transistors," Sci. Technol. Adv. Mater. Vol.11, no.4, 044305 (23pp), Aug 2010.

[9] Tze-Ching Fung Katsumi Abe, Hideya Kumomi and Jerzy Kanicki, "DC/AC Electrical Instability of R.F. Sputter Amorphous In-Ga-Zn-O TFTs", SID Symp. Dig. Tech., vol. 40, no.1, pp. 1117-1120, Jun 2009.

[10] Charlene Chen, Katsumi Abe and Hideya Kumomi and Jerzy Kanicki, "a-InGaZnO thin-film transistors for AMOLEDs: Electrical stability and pixel-circuit simulation.” J. Soc. Inf. Disp. Vol. 17, no.6, pp. 525-534, Jun 2009.

[11] Charlene Chen, Katsumi Abe, Tze-Ching Fung, Hideya Kumomi and Jerzy Kanicki, "Amorphous In-Ga-Zn-O Thin Film Transistor Current-Scaling Pixel Electrode Circuit for Active-Matrix Organic Light-Emitting Displays,” Jpn. J. Appl. Phys. Vol. 48, 03B025 (7pp) Mar 2009.

[12] Y. Toyota, M. Matsumura, M. Hatano, T. Shiba, M. Ohkura, "A new study on the degradation mechanism in low-temperature p-channel polycrystalline silicon TFTs under dynamic stress," IEEE Trans. Electron Devices, vol. 53,no. 9, pp. 2280-2286, Sept 2006.

[13] Michael S. Shur, Holly C. Slade, Mark D. Jacunski, Albert A. Owusu and Trond Ytterdal, "SPICE Models for Amorphous Silicon and Polysilicon Thin Film Transistors," J. Electrochem. Soc., vol.144, no. 8, pp. 2833-2839, Aug 1997. 